\title{
THE SURGICAL TREATMENT OF PULMONARY TUBER- CULOSIS IN CHILDHOOD AND ADOLESCENCE
}

\author{
BY \\ D. W. HUISH \\ From the Regional Thoracic Surgical Centre, Dundee
}

(RECEIVED FOR PUBLICATION OCTOBER 17, 1955)

Pulmonary tuberculosis as seen in childhood and adolescence presents many interesting problems to the physician and to the pathologist. An extensive literature has grown around the subject expressing conflicting views upon the pathogenesis, natural history, diagnosis, and treatment of the disease. The discussion has been confused by differences in terminology and by the different sources of the authors' material. Treatment of the disease has aroused no less controversy than its other aspects, but, in contrast to the numerous reports on the efficacy or otherwise of medical measures, surgical treatment has received little attention in the literature. Collapse therapy in the form of artificial pneumothorax or pneumoperitoneum has long been employed in children, but I have been able to trace only 11 papers describing series of planned major surgical interventions for pulmonary tuberculosis in children and adolescents, namely, those of Levitin and Zelman (1950), Botelho, Chapchap, Pereira, and Cordeiro (1951), Ross (1951), Métras (1952), Dillwyn Thomas (1952), Rubin and Mishkin (1952), Vojtek and Sery (1952), Giraud and Métras (1953), Liaras and Houel (1953), Boyd and Wilkinson (1954), and Giraud, Métras, Grégoire, Longefait, and Hartung (1954).

Isolated cases have been described by various authors (Vossschulte, 1951 ; Legrand, Razemon, Masure, and Soots, 1952), and incidental references have been made in papers concerning the surgical treatment of pulmonary tuberculosis in adults.

It is intended in this paper to review 29 cases of pulmonary tuberculosis in children under the age of 16 years treated at the Regional Thoracic Surgical Centre, Dundee, during the five years from December, 1949, to December, 1954, and to survey the literature.

\section{MATERIAL AND CLASSIFICATION}

The 29 cases were referred to the Centre by several chest physicians and paediatricians as indi- vidual problems and not as part of a programme for treating such cases by any particular method. All the children were of the white race, all being resident in the eastern part of central Scotland, coming from both urban and rural areas. They derive from a population of some 500,000 people, although this figure is only approximate, as the area served by the Centre has been modified during the period of this study. They represent, of course, only a tiny proportion of the cases of pulmonary tuberculosis in children in the area. Out of 208 cases admitted to the Children's Tuberculosis Unit in Dundee, only 11 were referred for surgery (Ritchie, personal communication).

Classification of the cases has been based on the simple system propounded by Bentley, Grzybowski, and Benjamin (1954). These authors found that on a clinical basis most cases could b? divided into two main types: (I) primary pulmonary tuberculosis; (II) chronic pulmonary tuberculosis. Primary pulmonary tuberculosis they subdivided into six groups.

Such terms as " post-primary," "epituberculosis," "reinfection type," and "tertiary type" were abandoned as having come to be used by different observers to describe too many diverse conditions. The term "chronic pulmonary tuberculosis" included all cases with the type of disease that is commonly encountered in adults, and for which such terms as "adult type," "reinfection type," "tertiary tuberculosis" are used.

This classification has been modified slightly for the purpose of the present series, as not all the subdivisions of primary pulmonary tuberculosis present indications for surgical treatment. The cases in this series were drawn mainly from these authors' Group 3 (primary pulmonary tuberculosis with lobar or segmental lesions) and Group 7 (chronic pulmonary tuberculosis).

The classification employed is thus as follows: "primary pulmonary tuberculosis" (a) with segmental or lobar lesions, $(b)$ with segmental or 


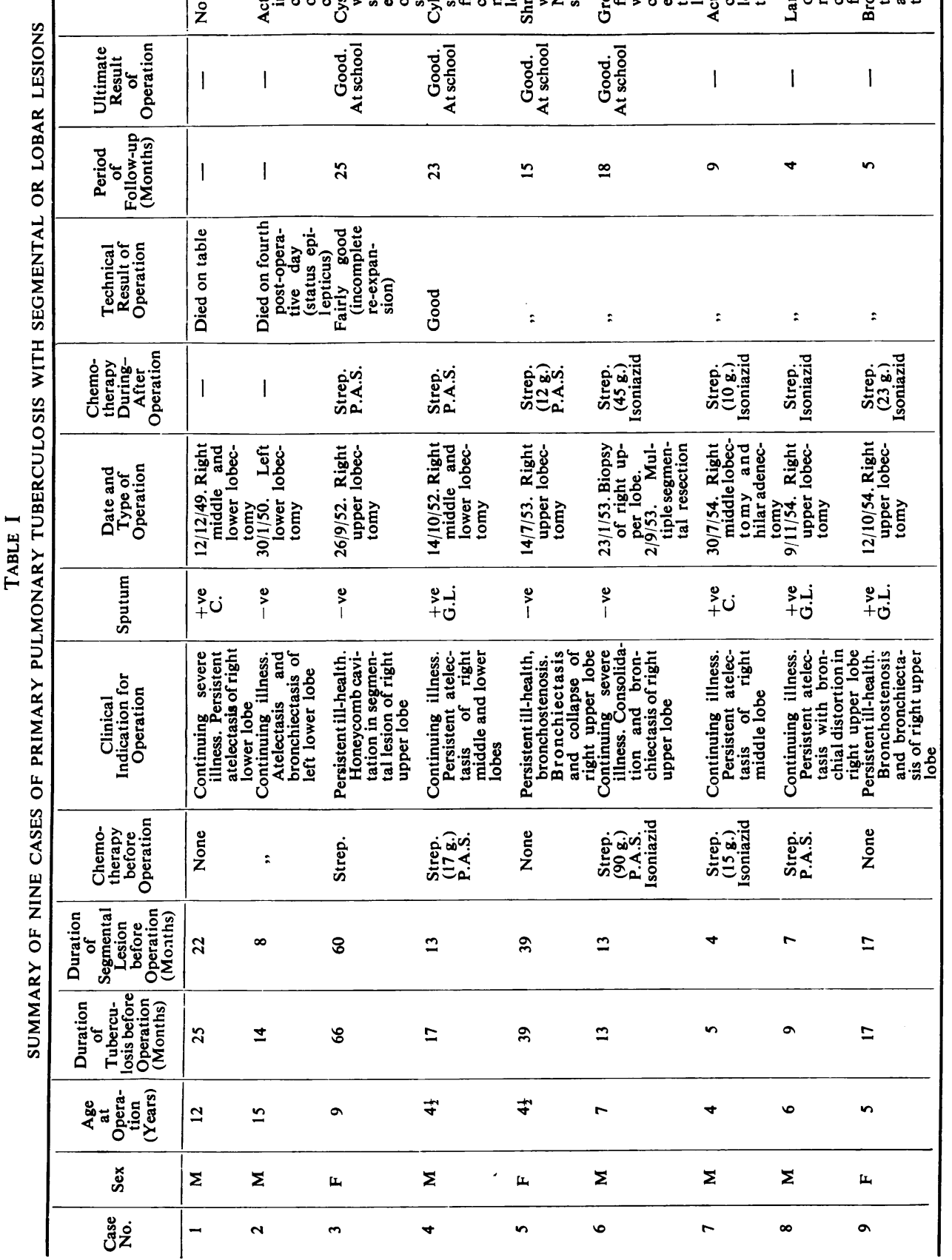

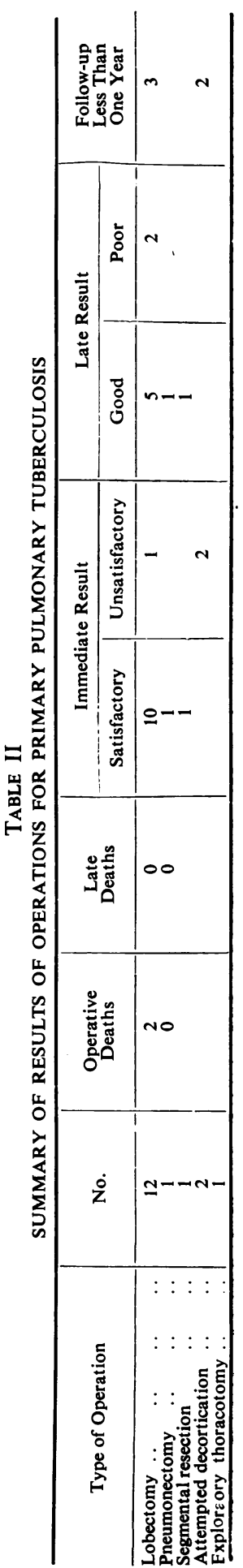

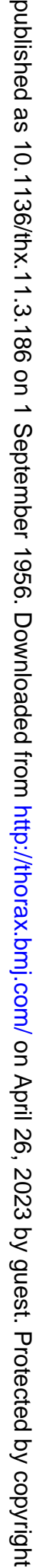


lobar lesions causing symptomatic bronchiectasis, (c) with primary cavitation, (d) with tuberculous pleurisy, (e) resulting in chronic fibroid lung, and "chronic pulmonary tuberculosis."

During what was often a long clinical course several of the cases showed other manifestations of tuberculosis; for instance Cases 6 and 8 had lesions in other parts of the body resulting from haematogenous dissemination; Case 1 had a pleural effusion at one stage. The cases have been classified according to the main presenting feature when referred for surgery.

\section{DESCRIPTION OF CASES}

\section{Primary Pulmonary Tuberculosis}

Fifteen children were operated upon for various manifestations of this stage of the disease, their ages ranging from 4 to 15 years. Seventeen operations were performed; the results are summarized in Table II.

(a) With Segmental or Lobar Lesions.-The nine cases in this group are summarized in Table I. It will be observed that 10 operations were performed upon the nine children, viz., eight lobectomies, one exploratory thoracotomy, and one double segmental resection. There were two operative deaths, but no late deaths. Of the seven survivors, four have been followed for over a year; all obtained a good result. The three cases followed for shorter periods have all obtained a good immediate result.

The fatal outcome in Case 1 was due to cardiac arrest during the operation. In Case 2 the patient passed into status epilepticus 48 hours after the operation; there was no response to treatment. The patient was a known epileptic, having suffered characteristic major episodes for several years.

The courses of two typical cases in this group (Nos. 4 and 8) are illustrated in Figs. 1 to 8.

(b) With Segmental or Lobar Lesions CausING SyMPtomatic Bronchiectasis. - Two cases of this type were encountered, the clinical picture being dominated by infected bronchiectasis. Both were treated by lobectomy. The result was good in one case but not in the other. The latter case illustrates the serious prolonged course which primary tuberculosis may follow.

Case 10.- In 1950 a boy, then aged 11, developed cough, dyspnoea, and loss of weight. In July, 1950, he had a haemoptysis, and a radiograph showed mottling at the left base. The sputum contained tubercle bacilli. He was admitted to hospital in September, 1950. Radiography now showed complete atelectasis of the left lower lobe with enlarge- ment of the left hilar lymph nodes. The Mantoux test was positive. Tubercle bacilli were not found in the sputum. Conservative treatment during the next 18 months produced no improvement, and productive cough persisted. Bronchography in March, 1952, showed gross bronchiectasis and contraction in the left lower lobe. Left thoracotomy was performed in April, 1952, and a mass of caseating lymph nodes was found in the hilum penetrating the wall of the lower lobe bronchus. The small fleshy lower lobe was removed by the dissection technique. The postoperative course was uneventful. In March, 1955, he was reported fit and well, at work. Radiographs showed both lung fields clear. The lobe removed was reported to show chronic suppurative bronchiectasis with diffuse interstitial fibrosis and contraction of the lobe, and no evidence of tuberculosis.

Case 11.-The mother of a boy, then aged 4, developed pulmonary tuberculosis in 1948 . During the next two years the boy developed severe cough with purulent sputum. A radiograph in August, 1950, when he was aged 6 , showed bilateral enlargement of the hilar lymph nodes. Bronchography showed no gross bronchiectasis. The Mantoux test was positive. In 1953, after he had had a left-sided pneumonia, bronchography revealed bronchiectasis affecting both lower lobes, the middle lobe, and the lingula. A calcifying primary focus was noted in the left upper lobe. Left lower lobectomy and lingulectomy in June, 1953, at the age of 9 produced only temporary improvement, and the grossly affected right middle lobe was removed in May, 1954. In July, 1954, he became acutely ill with signs of atelectasis in the left lung, but recovered on conservative treatment. In September, 1954, he became ill again, and radiographs showed massive enlargement of the left hilar lymph nodes with atelectasis of the remaining left upper lobe. At bronchoscopy the bronchus was compressed, oedematous, and filled with pus, from which tubercle bacilli were cultured. He was transferred to a medical ward for long-term chemotherapy.

(c) With Primary Cavitation.-Only one undoubted case was encountered showing both the clinical and histological characteristics of "primary" cavitation. The result of lobectomy was excellent.

Case 12.-A girl aged 3 had been in contact with several adult relatives with pulmonary tuberculosis, and in May, 1951, radiography showed a primary focus in the left upper lobe. Shortly afterwards she became ill and developed phlyctenular conjunctivitis. The Mantoux test was positive. She was admitted to hospital, where her general condition improved with bed rest. Serial radiographs showed little change until November, 1951, when a central cavity appeared in the primary focus, and increased in size despite chemotherapy. She was referred for surgery in September, 1952, at the age of 4 . Radiographs showed a medium-sized cavity in the left upper lobe with a thick ring of denser tissue round it. The left hilar 


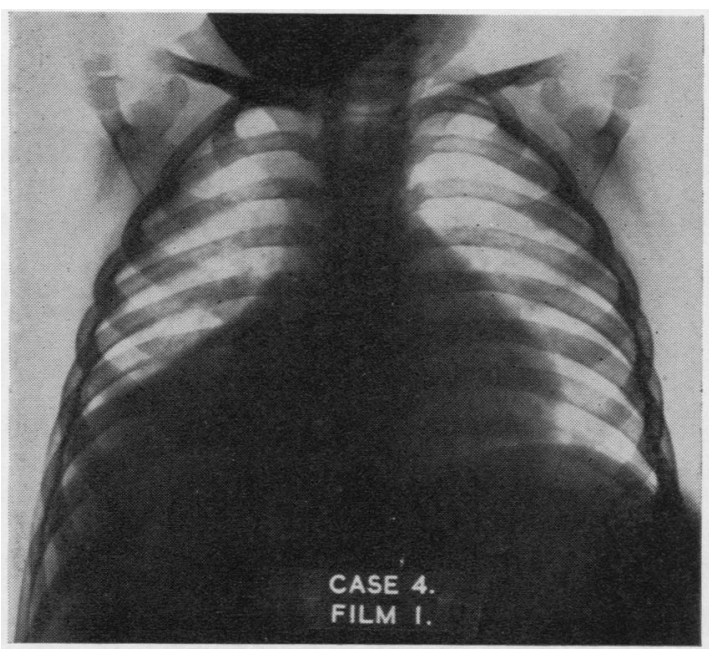

FIG. 1.-Case 4, 13 months before operation, showing collapse consolidation of the right middle and lower lobes.

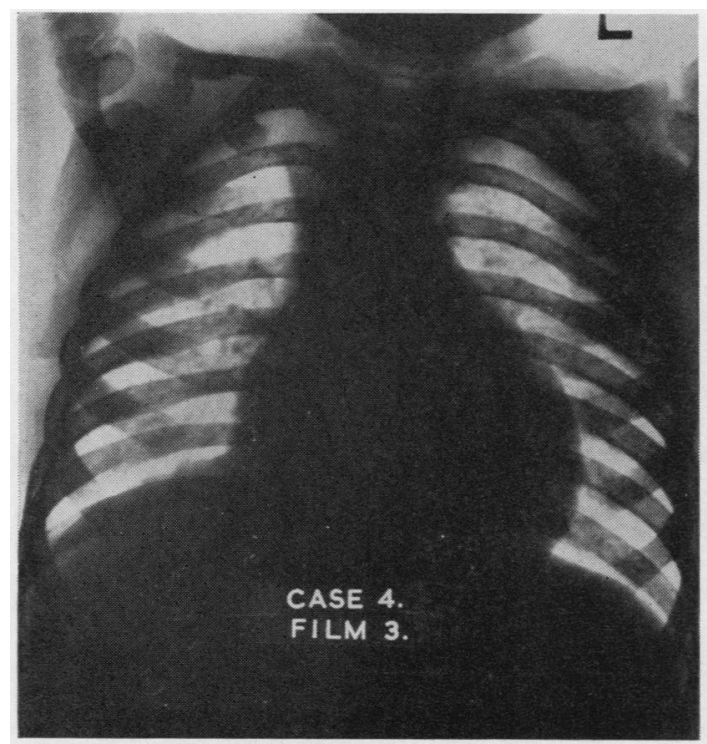

Fig. 3.-Case 4, two months after right middle and lower lobectomy.

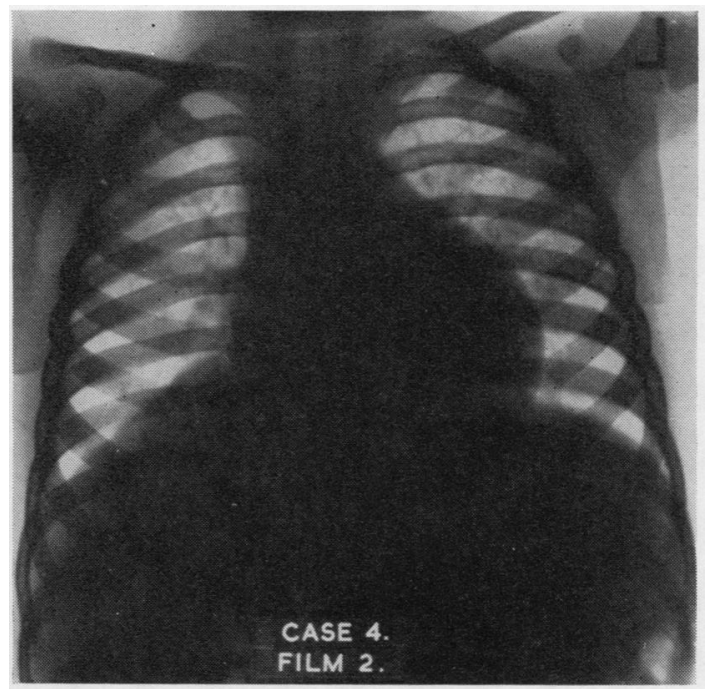

Fig. 2.-Case 4, immediately before operation, showing persistence of the lobar lesion.

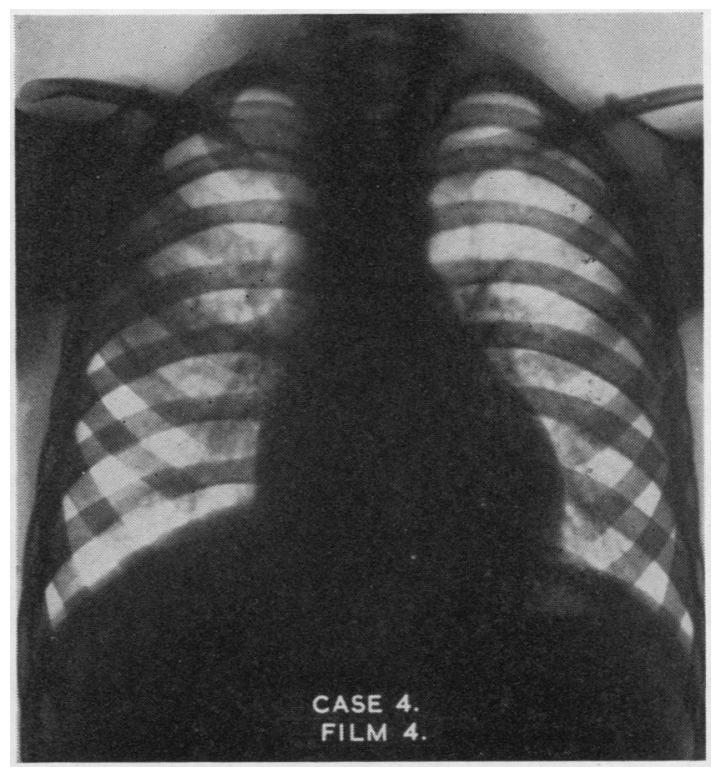

Fig. 4.-Case 4, 23 months after operation. 


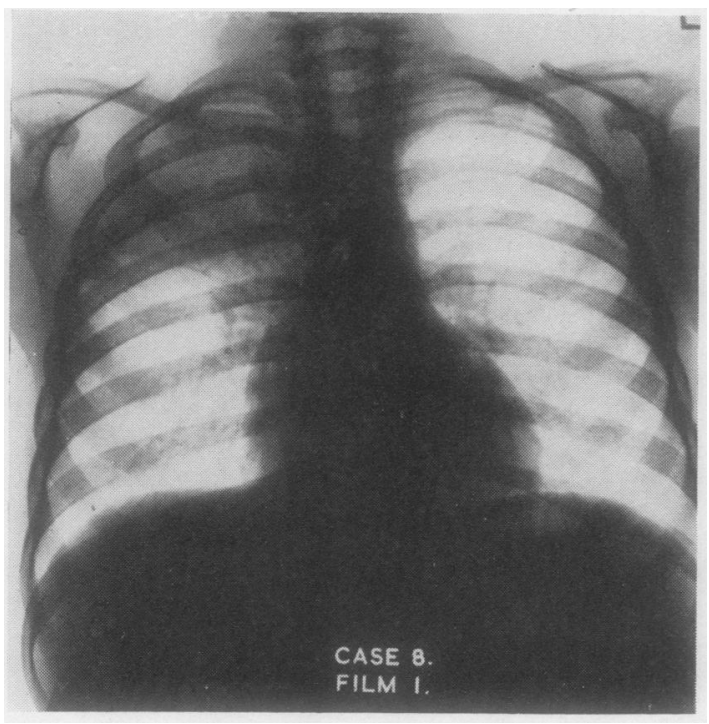

Fig. 5.-Case 8, seven months before operation, showing collapse consolidation of the right upper lobe.

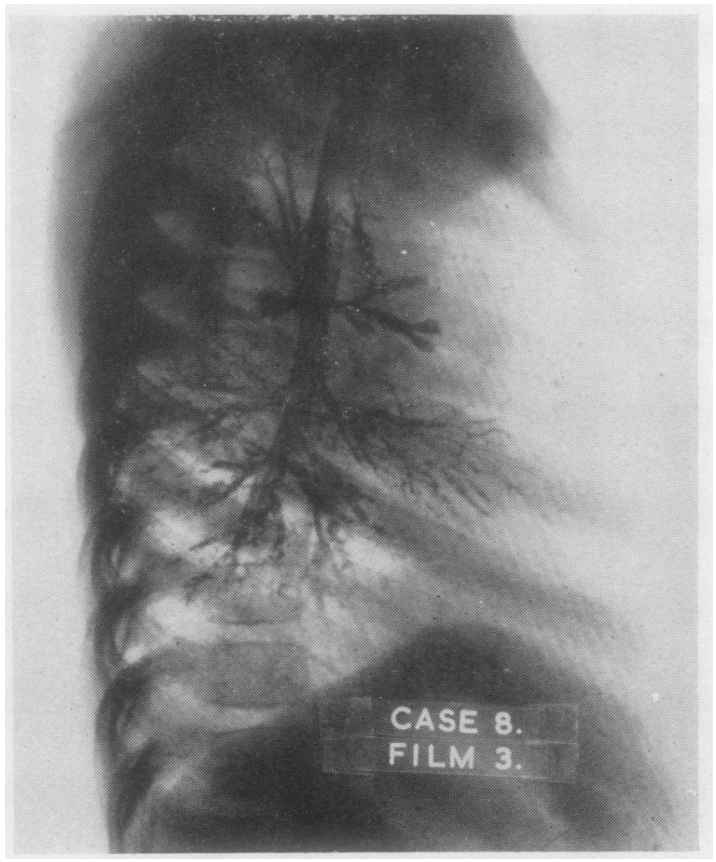

FIG. 7.-Case 8, pre-operative bronchogram, showing blocking of the posterior segment of the right upper lobe with bronchiectasis and distortion of the other two segments.

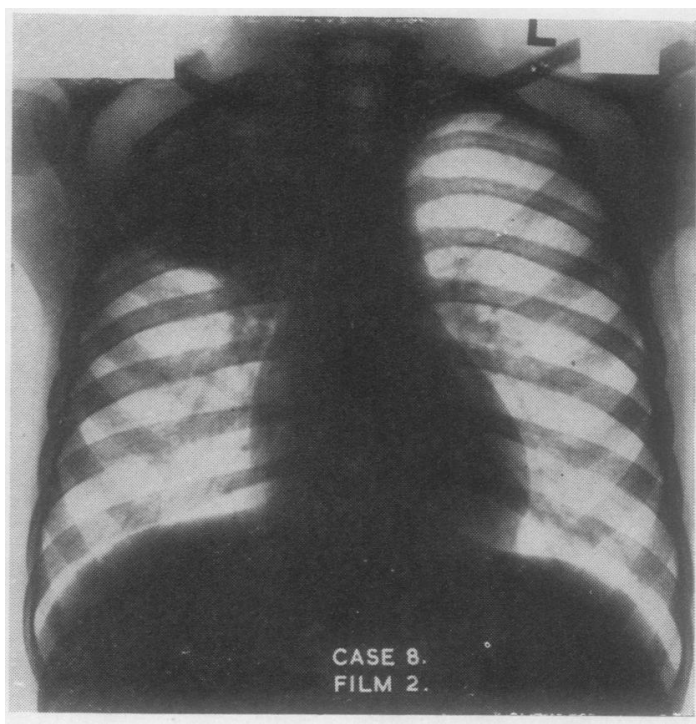

FIG. 6.-Case 8, one month before operation, showing persistence of the lobar lesion.

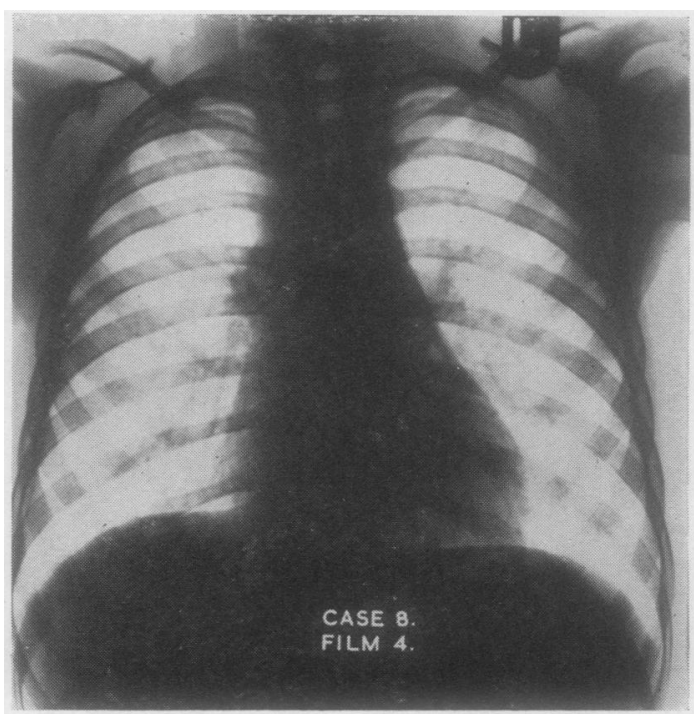

Fig. 8.-Case 8, two months after right upper lobectomy. 


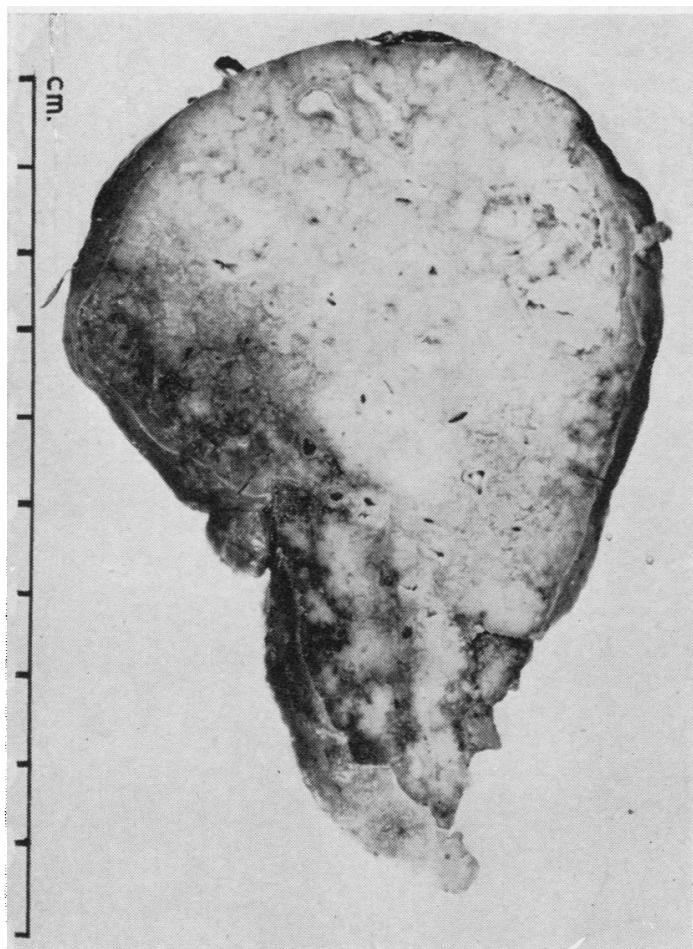

Fig. 9.-Case 8, operation specimen, showing extensive areas of caseation and innumerable tuberculous lesions replacing the greater part of the lung tissue.

lymph nodes were enlarged. Left upper lobectomy was performed in October, 1952, by the mass ligation technique, dissection being prevented by a mass of haemorrhagic lymph nodes in the hilum. The postoperative course was uneventful. In March, 1955, she was reported well and attending school, and radiography showed normal lung fields. The lobe removed was reported to show a tuberculous cavity, $1.5 \mathrm{~cm}$. in diameter, with active spreading tuberculous granulation tissue around the margins of the massive caseation, and many small caseous foci in the remaining lung tissue, relatively inactive with fibrous capsules.

(d) With Tuberculous Pleurisy.-Sero-fibrinous pleurisy is a common feature of primary pulmonary tuberculosis, but it is rare for the pleurisy to become purulent or caseous. One case of this rare type was encountered.

Case 13.-A boy aged 12 was admitted to hospital in June, 1953, acutely ill with a large pleural effusion on the left side. The fluid was serous and yielded human type tubercle bacilli on culture. He was treated by chemotherapy and bed rest. By March, 1954, his general condition had greatly improved, but radiographs now showed gross pleural thickening with contraction of the left lung. Bronchography showed no evidence of bronchiectasis. Thoracotomy was performed in April, 1954, with the intention of decorticating the left lung. The pleural space was found completely obliterated, and there was a thick layer of fibrous granulation tissue over the lung up to $2 \mathrm{~cm}$. thick in places. This layer was frankly caseous and contained pockets of pus. The operation had to be abandoned. He made a good recovery from the operation and returned to the medical wards. In December, 1954, he was still in hospital, and the pleural thickening appeared to be clearing after further chemotherapy. The excised tissue was reported to show fibrous and granulomatous tissue containing many active caseating foci.

(e) Resulting in Chronic Fibroid Lung.-Two cases of this comparatively rare condition were encountered. In both cases a whole lung was involved, resulting in fibrous contraction of the lung with bronchiectasis, following the pattern described by Bentley and others (1954). One was cured by pneumonectomy.

Case 14.-A girl, then aged 4, became ill in January. 1951, while living abroad. Radiographs showed enlargement of the left hilar lymph nodes with complete atelectasis of the left lung and a small pleural effusion. The Mantoux test was positive. Treated by bed rest, she improved rapidly and the lung became re-aerated. In April, 1951, the left lung was aga:n found to be collapsed. Tubercle bacilli were found by gastric lavage. She returned to Scotland, and in June, 1951, again became ill with complete opacity of the left lung and extreme mediastinal shift to the left. In December, 1951, she was admitted to hospital and received chemotherapy. Her general condition improved, and she was referred for surgery in February, 1953, at the age of 6 because of persistent productive cough and poor general health. $\mathrm{A}$ radiograph showed enlargement of the left hilar lymph nodes with contraction of the left lung. Bronchography showed gross cystic bronchiectasis affecting all segments of the left lung. In May, 1953, left pneumonectomy was performed by dissection technique; many large lymph nodes were encountered in the hilum, especially in the subcarinal group. The post-operative course was uneventful. In March, 1955, she was reported to be well and attending school, free from symptoms and with no respiratory disability. The lung removed was reported to show cystic bronchiectasis with fibrosis and low-grade inflammatory changes, with no evidence of tuberculosis in the sections examined. The hilar nodes showed caseating tuberculosis.

Case 15.-A boy, then aged 3, became acutely ill in October, 1947. Radiography showed a primary complex in the left upper lobe with enlargement of the left hilar lymph nodes. The Mantoux test was positive, and tubercle bacilli were found on gastric lavage. Admitted to hospital, he was treated by bed rest. In December, 1947, the left lung became completely atelectatic, and did not re-aerate until March, 1949. He was discharged home in July, 1949 . He remained well, but by 1953 it became noticeable that the left side of the chest was smaller than the right and 
覆

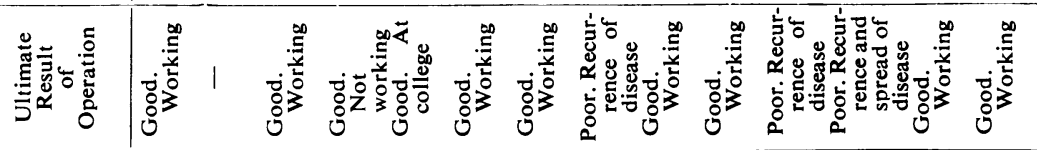

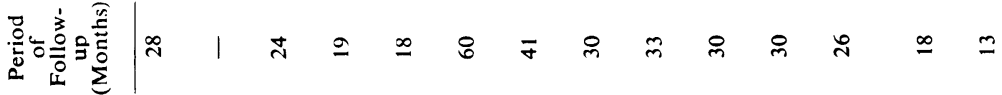

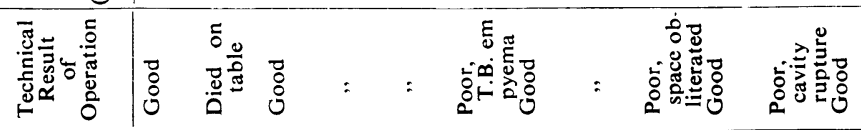

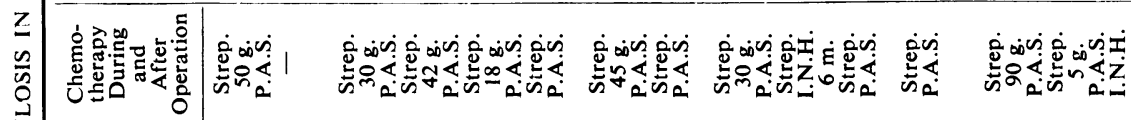

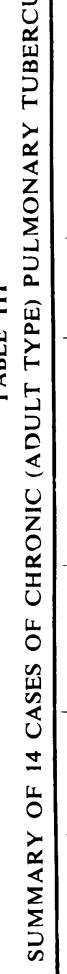

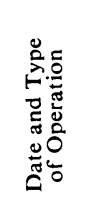

|

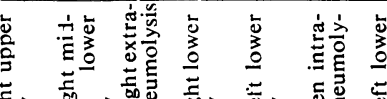

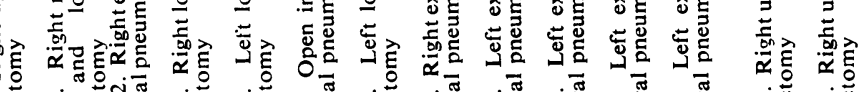

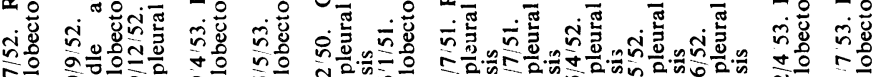

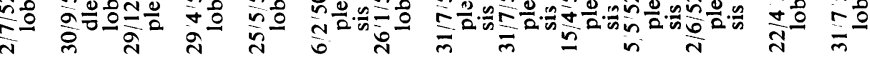

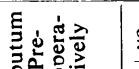

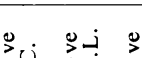

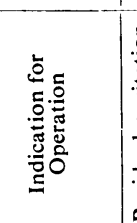

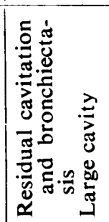

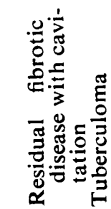
into

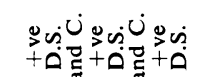
ن فำ

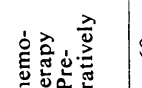

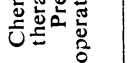
$\dot{\infty} \dot{\infty} \dot{\infty}$

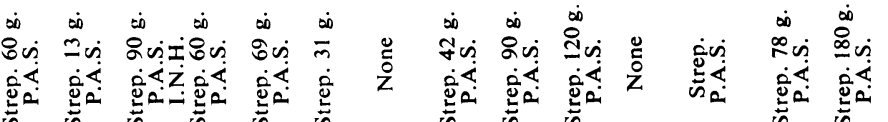

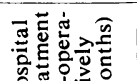

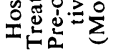

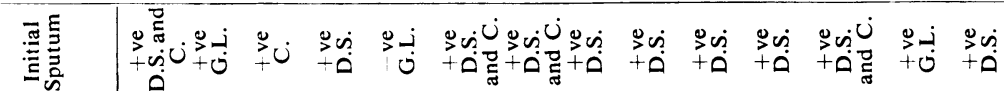

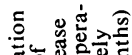

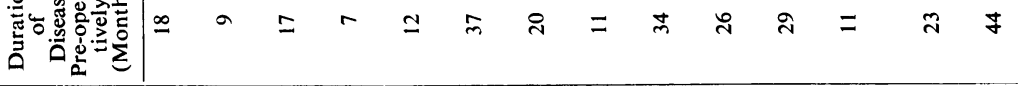

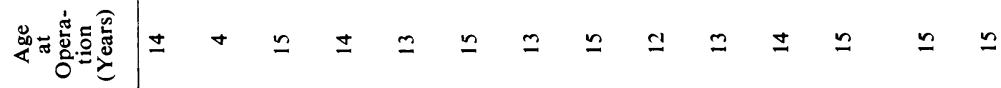

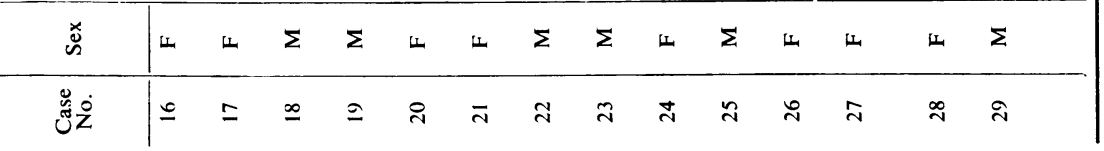




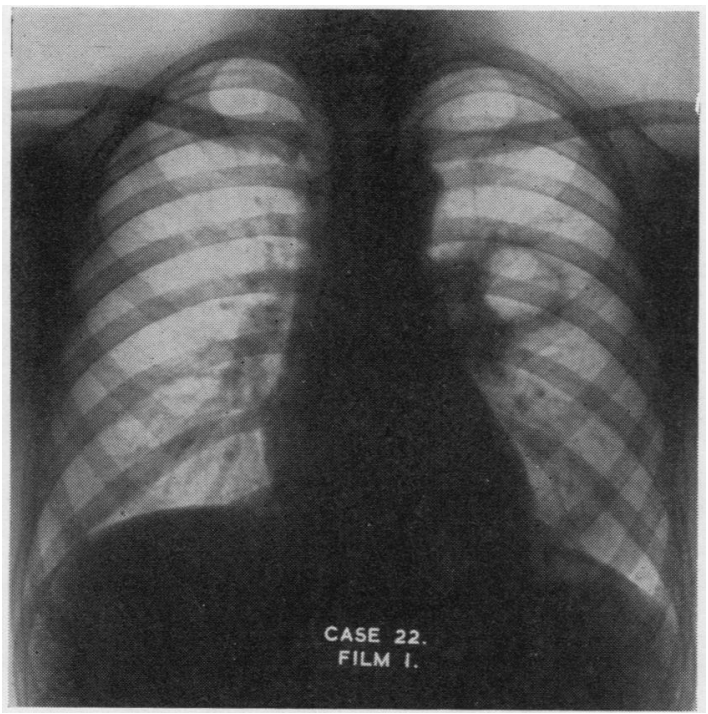

FIG. 10.-Case 22, pre-operative film showing large chronic cavity in apex of left lower lobe.

expanding less. Radiography showed contraction of the left lung. Bronchography showed distortion and narrowing of the left main bronchus, with some bronchiectasis in the left upper lobe. In May, 1954, thoracotomy was undertaken, with the intention of decorticating the lung, removing any glands around the left bronchus, and performing upper lobectomy

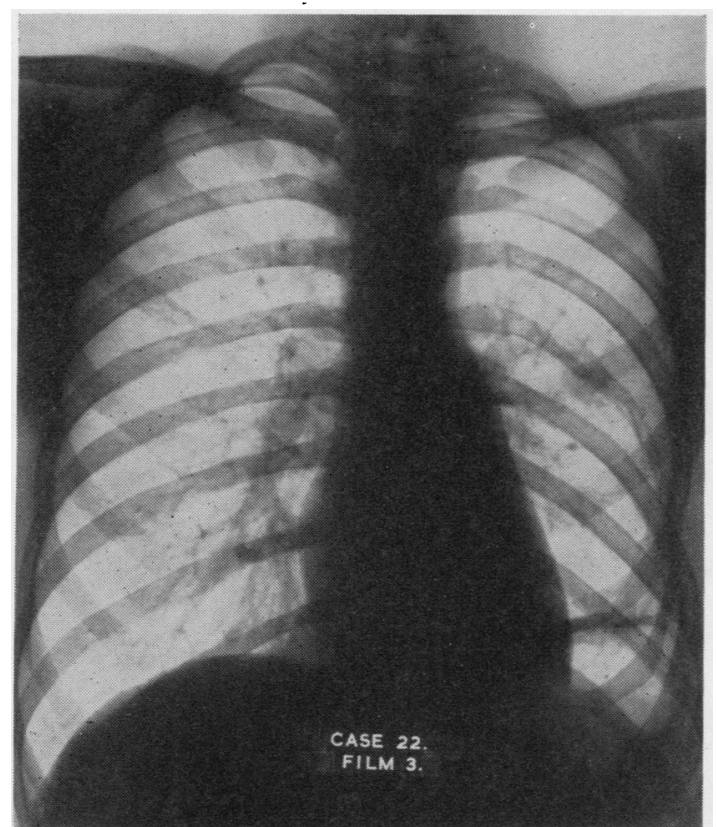

FIG. 11.-Case 22, three years after left lower lobectomy; the left lung field is clear, the diaphragm remains elevated. if necessary ; but the pleural space was found obliterated by dense adhesions, many of which contained large blood vessels carrying anastomoses between the chest wall and pleura. Operation had to be abandoned because of the vascularity of the adhesions. Postoperatively a small haemothorax developed, and was dealt with by aspiration. In December, 1954, the preoperative picture was unchanged.

\section{Chronic Pulmonary Tuberculosis}

Fourteen patients were operated upon for various manifestations of this disease. The cases are summarized briefly in Table III. Five (Nos. 16 to 20) arose in close clinical relation to a primary infection, and the other nine showed the type of disease usually seen in young adults. The clinical course in all of them indicated a severe form of tuberculosis. Fourteen operations were performed on these fourteen patients, the results being summarized in Table IV. The one operative death (Case 17) was due to massive collapse of the other lung (confirmed by necropsy). The course of a typical case (No. 22) is illustrated in Figs. 10 and 11.

\section{DISCUSSION}

\section{Primary Pulmonary Tuberculosis}

The role of surgery in the treatment of any disease should be securely based on a study of the pathology. This is no less true of primary pulmonary tuberculosis, but here unfortunately the pathology is by no means clear, and as a result clinical interpretations tend to be based on personal views. The available evidence has been ably marshalled by Rich (1951).

At the present stage of our knowledge simple primary tuberculosis presents no indication for surgery. It is the complications which are of surgical interest. The focus in the lung may spread locally as a destructive caseous pneumonia, resulting in primary cavitation. One of the commonest complications, and the one of greatest surgical interest, is the appearance of a segmental or lobar lesion, first described by Eliasberg and Neuland in 1920 under the title of "epituberculous infiltration."

This type of lesion may involve one or more bronchopulmonary segments, the whole of a lobe, or occasionally a whole lung. There is general agreement (Rich, 1951 ; Adler and Richards, 1953 ; Bentley and others, 1954) that the general course of these lesions is benign, the shadow persisting for some time and then slowly clearing over a period of months or even years. Opportunities for necropsy examination are rare, and conflicting 
views have been expressed by authors making isolated observations on different stages of the disease. The main views are that these segmental lesions represent (1) a tuberculous pneumonia, or (2) non-tuberculous consolidation, or (3) atelectasis resulting from bronchial obstruction. In recent years the use of the bronchoscope to investigate these lesions during life, particularly by the Continental school (Laff, Hurst, and Robinson, 1951 ; Jeune, Bertoye, Mounier-Kuhn, and Beraud, 1952), has led to a greater appreciation of the important role played by the mediastinal lymph nodes. When these nodes become enlarged with gross caseation during primary infection, they may compress a main or segmental bronchus, and may then progressively infiltrate the wall of the bronchus and finally perforate into its lumen, discharging a mass of caseous material which further obstructs the bronchus. Serial bronchoscopic examinations of individual cases have clearly demonstrated this sequence of events (Daly, Brown, Lincoln, and Wilking, 1952 ; Valledor and Navarrete, 1952). The appearances have been well described in detail by Podvinec and Erak (1951), who have also drawn attention to the significance of reflex congestion and swelling of the bronchial mucosa in causing bronchial obstruction. The anatomy of the mediastinal lymph nodes and their response to primary tuberculous infection have been carefully investigated by Engel (1947). He showed that the infection of the regional nodes draining the primary lung focus is of less account than the infection of the right tracheo-bronchial and bifurcation nodes which form a second line of defence. As a result of the anatomical disposition of these nodes the right main bronchus is particularly liable to compression and perforation.

These observations may be correlated with the experimental work on animals reviewed by Rich (1951). He concluded that all three factors mentioned above, tuberculous pneumonia, non-tuberculous consolidation, and atelectasis, may be concerned in the production of bronchopulmonary lesions ("epituberculosis"), depending on the number of bacilli, living or dead, introduced into the bronchus by lymph-node perforation, and the degree of compression or internal obstruction of the bronchus. In the majority of cases atelectasis and non-tuberculous consolidation are probably the main factors. Some of the French authors believe that the non-tuberculous consolidation results from reflex exudation into the alveoli following disturbance to the nerves and vessels of the lung by the enlargement of the hilar glands (Jeune and others, 1952), but they give little evidence to support their views.

While the general course of bronchopulmonary lesions of this type is accepted by all observers as being benign, it should be noted that this is not necessarily so in any particular case. Out of 169 patients with such lesions observed by Bentley and others (1954), four died, two from tuberculous meningitis, one of massive bronchogenic spread throughout a whole lung, and one of chronic pulmonary tuberculosis. Twenty-threz developed tuberculous complications but survived. The total experience in regard to both deaths and complications was somewhat worse in this group as compared with simple primary tuberculosis.

In recent years increasing interest has been manifested in the ultimate fate of the affected part of the lung, the discussion centring around the development of bronchiectasis and bronchostenosis. In this connexion it would seem advisable to draw a distinction between the anatomical lesion as demonstrated by bronchography or the examination of resection specimens, and the clinical disease. The anatomical lesion seems to be the more often found the more often it is sought. Hutchison (1951) studied 34 cases of primary pulmonary tuberculosis with bronchial occlusion for periods varying from six months to 10 years ; 18 developed anatomical bronchiectasis, four developed bronchostenosis, and in only eight cases did the bronchograms appear completely normal. Valledor and Navarrete (1952) studied 250 cases of primary pulmonary tuberculosis over a period of 15 years. Anatomical bronchiectasis of varying degree was found in about $70 \%$. Williams and Anderson (1953) studied 40 cases of primarv pulmonary tuberculosis w:th segmental lesions ; 32 developed anatomical bronchiectasis and 10 of these had bronchostenosis. As regards symptomatic bronchiectasis the position is very different : in all the reported investigations the incidence of this condition was low. Bentley and others (1954) encountered only 10 cases of symptomatic bronchiectasis in following up 169 patients with segmental lesions. All 10 cases involved the middle or lower lobes. Valledor and Navarre+e (1952) found that the majority of their cases of anatomical bronchiectasis were dry and uncomplicated, but occasionally observed severe svmptoms causing invalidism. Dickey (1952) described two cases of severe symptomatic bronchiectasis of the left lower lobe following primary lobar lesions. Both cases had positive sputum. He also described a case with recurrent symptoms due to bronchiectasis of an upper lobe. There is general 
agreement that symptomatic bronchiectasis usually follows lesions of the lower lobe, especially on the right side. In most of the reported studies the follow-up was not continued for more than a few years. The question of a connexion between anatomical bronchiectasis following primary pulmonary tuberculosis and symptomatic bronchiectasis in adult life has not been settled and requires more study. Brock (1950) described the so-called "middle lobe syndrome," a bronchostenosis and bronchiectasis of the middle lobe associated with evidence of previous tuberculosis of the hilar lymph nodes. Valledor and Navarrete (1952) after their extensive study believe that such a connexion probably exists.

Little has been published about the fate of the alveolar tissue of the affected segment. Contraction, fibrosis, and calcification are the main changes described. Adler and Richards (1953) studied the final radiographs of 66 cases with segmental lesions and concluded that $86 \%$ of these were left with residual collapse, collapseconsolidation, or atelectasis, involving at least one segment. Complete re-expansion seemed to be rare. Bentley and others (1954), also from radiological evidence, concluded that the most frequent end-result is a greatly contracted scarred re-aerated segment. Giraud and Métras (1953) also observed this phenomenon, and described the "ferme l'éventail" as a radiological sign of irreversible damage to the affected segment. The experimental work of Burke (1940) would suggest that histologically the end-result would be fibrosis and occasional scattered healed tubercles. That this hypothesis is correct is suggested by some of the resection specimens in the present series (Cases 3, 4,5 , and 9), and in the series of Boyd and Wilkinson (1954).

The experimental observation of Oppenheimer (1935), that definite tuberculous tissue is often still present after radiological clearing, may be of surgical importance. Stevens (1952) has observed by routine guinea-pig inoculation of gastric contents that a high proportion of these cases with segmental lesions continue to produce tubercle bacilli over a long period. Dijkstra (1952) has demonstrated the same phenomenon by means of frequent sputum culture over prolonged periods, and has demonstrated that it may persist long after the associated bronchial lesions have completely healed.

Should lymph-node compression or perforation result in complete obstruction of either main bronchus, the resulting lesion may involve a whole lung. The end-result in such cases has been described by Bentley and others (1954) as "chronic fibroid lung." The lung is destroyed by a large number of caseous foci resulting in gross contraction with bronchiectasis and sometimes cavitation. Severe symptomatic bronchiectasis often develops, and chronic pulmonary tuberculosis is liable to develop as puberty approaches. Many of the pneumonectomies recorded in children appear to have been done for lesions of this type (Botelho and others, 1951; Liaras and Houel, 1953). Case 14 of the present series is a good example.

Pleural effusion is probably the commonest complication of primary pulmonary tuberculosis, arising either by direct involvement of the pleura by the primary lung focus or lymph node, or by haematogenous spread. The effusion reabsorbs within a few months, generally leaving no trace beyond pleural adhesions and slight pleural thickening. Bentley and others (1954), however, in a study of 226 cases noted in a few instances considerable pleural thickening and contraction of the affected hemithorax.

On pathological grounds, therefore, indications for operation in primary pulmonary tuberculosis seem to arise in only a small proportion of cases in the following circumstances: (1) primary cavitation; (2) segmental or lobar lesions, where the disease process is a caseating tuberculous pneumonia, not responding to conservative treatment ; (3) bronchial compression or perforation by mediastinal lymph nodes at an early stage, either where the bronchial obstruction in itself is causing severe symptoms or where the resulting pulmonary lesion may be regarded as still reversible ; (4) disease of segmental or lobar extent due to involvement of main bronchi by pressure or erosion from enlarged lymph nodes, which has resulted in secondary infection and symptomatic bronchiectasis, either at an early or late stage ; (5) similar changes to (4), which have reached the stage of contraction, fibrosis, and anatomical bronchiectasis, but are still causing symptoms or still excreting tubercle bacilli; (6) lesions involving a whole lung, at the late and irreversible stage of chronic fibroid lung; (7) pleural thickening after effusion, causing gross interference with the function of the affected lung.

Primary cavitation is a rare condition which should be treated exactly as a tuberculous cavity in the adult lung (Levine, 1953). On this basis, should conservative measures fail to bring the condition under rapid control, removal of the cavity by resection would appear to be indicated. The treatment of adult cavitation by resection is 
now well established, and resection of primary cavities in children has given good results in the few cases which have come to operation (Rubin and Mishkin, 1952). The excellent result of lobectomy in Case 12 of the present series supports this view. While local resection of the cavity might seem attractive, the spreading nature of the lesion and the technical difficulties caused by the associated tuberculous hilar lymph nodes probably render lobectomy a safer and better operation.

The type of segmental or lobar lesion where the disease process is a caseating tuberculous pneumonia forms only a small proportion of the total, but is probably a frequent cause of the more severe types of clinical illness. Dillwyn Thomas (1952) reported 27 cases of resection of a lobe or segment for primary pulmonary tuberculosis. In 26 of these the resection specimens showed areas of caseating tuberculosis, some very extensive. In all of these extensive cases a gland was found perforating into the draining bronchus. The only cases in the present series showing these extensive changes were Cases 2 and 8 (see Fig. 9). In both of these the clinical illness was severe, Case 8 originally presenting with acute miliary tuberculosis. While there is no doubt that such cases frequently resolve (Stevens, 1952), and the difficulties of diagnosis preclude an accurate estimation of the mortality rate, they often give rise to considerable anxiety, and some do not do well. It may be that as antibacterial drugs cure more patients with miliary tuberculosis and meningitis more cases of this type will be seen. Where the course suggests progression or delay in regression surgical resection, usually lobectomy, seems justifiable. Of 27 lobectomies, Dillwyn Thomas lost only one patient, and the immediate results were good. In the present series one patient (Case 2) died of an extraneous cause, status epilepticus, but the other (Case 8) obtained a good immediate result.

The possibility of operative treatment of bronchial compression and obstruction at an early stage of the disease has attracted much attention in recent years. If successful, this might not only relieve the symptoms due to the bronchial changes but also reverse the associated pulmonary lesions. Where the pulmonary lesion is an actual tuberculous pneumonia, no treatment of the bronchial lesion is likely to have any effect, but in the more common types due to non-tuberculous consolidation and atelectasis good results might be expected. It may be possible to clear the bronchial lumen by aspiration or forceps extraction through the bronchoscope, and this method has been advocated by several authors. Carlens and Hellström
(1953) obtained satisfactory re-aeration of the affected lobe in six out of 13 cases in which the bronchial lumen could be cleared. Podvinec and Erak (1951) described cases in which great relief of symptoms and satisfactory re-aeration were attained, and others in which the radiological appearances showed only a temporary improvement. Hutchison (1951) noted little benefit from the procedure, an opinion shared by Bentley and others (1954), who observed no improvement in eight cases subjected to it.

Vossschulte (1951) described a case of acute respiratory distress in a boy of 14 , caused by perforation of tuberculous mediastinal lymph nodes into the lower end of the trachea. Conservative measures having failed, right thoracotomy was performed, and after division of the azygos vein a mass of tuberculous nodes was dissected out of the angle between the trachea and right upper lobe bronchus. The patient was completely relieved of his respiratory distress. Vossschulte drew attention to various operations of a similar type performed mostly on a mistaken diagnosis of mediastinal tumour, one as long ago as 1911. He suggested that operative removal of the nodes should be undertaken in all cases of perforation into the trachea and of opacity involving an entire lung. Dillwyn Thomas (1952) described 10 cases of hilar adenectomy performed without any other interference, as well as 27 cases where this procedure had been combined with resection of a lobe or segment. He gave few details of the criteria for selection of cases and little assessment of the results beyond describing these as satisfactory. Giraud and Métras (1953) also attempted to prevent the development of bronchiectasis and bronchostenosis by excision of lymph nodes and relieving the obstruction of the bronchus. They operated on three children, aged 17 months to $4 \frac{1}{2}$ years, excising lymph nodes in all three cases; in two the bronchus was also incised and its caseous, purulent contents removed. They regard the radiological sign of "closing the fan" (ferme l'éventail) as the most important indication of irreversible damage. The presence of slight cylindrical bronchiectasis in the affected lobe or segment they regard as an indication for prophylactic operation; once gross bronchiectasis is established the moment has passed. In careful post-operative studies of two of the cases they demonstrated complete clearing of the bronchostenosis, with only slight cylindrical bronchiectasis remaining in one case. Further experiences with this procedure are briefly described by Giraud and others (1954). 
A case would therefore seem to have been made out for operative intervention at an early stage ; this method attacks the cause of the trouble directly and preserves the lung tissue intact. In the present series hilar adenectomy was not employed as an isolated procedure but was once used in combination with lobectomy (Case 7). It should be noted that the method is not without its dangers and may be technically difficult. The nodes are very adherent to and may actually penetrate the great vessels of the hilum. Dillwyn Thomas (1952) lost one case because of haemorrhage from a pulmonary artery, and Giraud and Métras (1953) encountered difficulty in preserving the bronchial arteries.

The development of symptomatic bronchiectasis after a segmental or lobar lesion is the clearest indication for operative intervention in primary pulmonary tuberculosis. The symptoms are of course due to secondary pyogenic infection, and, as stated above, this is comparatively uncommon. Dickey (1952) described two cases of this type treated by lobectomy, the affected lobe in each case being the left lower. The results were very satisfactory. In both cases the sputum, which had been positive, remained consistently negative after the operation. In the two cases in the present series (Cases 10 and 11), secondary infection supervened at an early stage. The result of lobectomy in one case was very good, but in the other (Case 11) the result was poor because of continued tuberculous activity in the mediastinal lymph nodes.

The operative removal of a pulmonary lesion which has reached the stage of contraction, fibrosis, and anatomical bronchiectasis depends not so much upon the pathological significance of these changes as upon the symptoms which are to be attributed to them. Most of the lesions of this type in the present series had reached this stage (Cases 3, 4, 5, and 9). Many patients with this type of lesion are clinically well, but some have symptoms. As already noted, Dickey (1952) described a case of this sort, and Bentley and others (1954) noted recurrent pneumonitis due to secondary infection in these scarred segments. In the present series the main symptom was persistent ill-health. In Case 3 this ill-health persisted six years after the onset of the segmental lesion. The improvement in health after lobectomy in all these cases was noteworthy. Boyd and Wilkinson (1954) report similar findings. The prolonged ill-health may be connected with the observation of Dijkstra (1952) that tubercle bacilli may be found persistently at this stage. This author described five cases of resection in late stages of primary pulmonary tuberculosis, the patients' ages ranging from 17 to 23 years. In one case the indication was primary cavitation. In two of the others persistent excretion of tubercle bacilli was proved to be due to a caseating primary focus within a segmental lesion. In one it was due to a lymph node perforating the middle lobe bronchus, and in the last case to stenosing tuberculous bronchitis affecting a single segmental bronchus. A relationship has been suggested (Bentley and others, 1954) between these "healed" segmental lesions and chronic pulmonary tuberculosis in later life. Were this to be proved, the case for resecting such lesions would be greatly strengthened. In the meantime the management of these cases rests on an indefinite and unsatisfactory basis.

Chronic fibroid lung might possibly be prevented by a wider application of lymph-node excision for relief of bronchial obstruction, but once developed it constitutes a clear indication for operation, usually in the form of pneumonectomy. The reported results of this procedure are uniformly good (Levitin and Zelman, 1950; Botelho and others, 1951 ; Liaras and Houel, 1953). In the one case of pneumonectomy in the present series (Case 14) the result was also very good. In children the dead space in the hemithorax is rapidly obliterated and there is no respiratory disability. The one contraindication to pneumonectomy is the presence of a lesion in the other lung. Lowys and Joly (1951) pointed out that thoracoplasty has a place when this situation is met with in older children, provided that suppurating bronchiectasis has not developed. They employed eight-rib thoracoplasty in two children aged 12 and 13 years, with minimal resulting deformity. They emphasize the importance of preserving the transverse processes and of avoiding damage to the lateral vertebral muscles and ligaments.

I have found no record of decortication being employed to deal with the end-results of primary pleural effusion in children, but the operation has a well-established place in adults (Quinlan, Schaffner, and Hiltz, 1952; Semb, 1954) and there seem to be even more cogent reasons for its application to children. Impaired function of a lung means much to a growing child, and may result in considerable deformity of the thorax from asymmetrical development. Again, the operation would apply to only the small proportion of cases in which gross pleural thickening and contraction of the hemithorax were appearing. Both cases (Cases 13 and 15) attempted in this series failed, in one 
because of unexpected activity in the tuberculous process in the pleura, and in the other because of the degree of underlying lung damage. In more favourable cases good results might be obtained, as in adults.

The great practical difficulty in deciding upon the indications for surgical treatment in any particular case of primary pulmonary tuberculosis is that of correlating the clinical and radiological findings with the pathological stage of the disease. From the large numbers of cases which pursue a benign course those with certain unusual trends must be selected.

The clinical features of the disease are on the whole variable and unreliable. Continuous severe illness with a bronchopulmonary lesion suggests a caseous tuberculous pneumonia. The presence of a characteristic, persistent, irritable cough similar in character to whooping-cough suggests bronchial perforation (Podvinec and Erak, 1951). Symptomatic bronchiectasis is easily recognized. Persistent ill-health is usually the only presenting feature. Physical examination is of little help.

Assessment must depend mainly on serial radiological examinations. Primary cavitation is thus easily recognized. It is in the interpretation of segmental or lobar opacities that uncertainty reigns. Some authors, for example, Adler and Richards (1953), claim that collapse, consolidation, and atelectasis can be distinguished radiologically at an early stage, depending on the area of the opacity compared with the estimated area of the normal segment or lobe. Others, for example, Bentley and others (1954), find themselves unable to make this distinction and regard the radiological differences as due to differing stages of the disease process. The previous consideration of the mixed nature of the underlying pathology supports this view. Continental authors emphasize the significance of the Jakobson sign in indicating atelectasis. The appearance of contraction of the shadow with re-aeration indicates that healing has taken place, but the contraction (the "closing of the fan" of the French authors) indicates also irreversible damage to the lobe. The length of time required for re-aeration is very variable, but the process generally takes several months. As a working plan it is suggested that patients showing unaltered or increasing segmental opacity with continuing illness, and those showing contraction of the opacity, warrant further investigation by bronchoscopy and bronchography. Those at a late stage in ill-health, with or without much change in the radiograph, warrant bronchography and repeated cultures of sputum or gastric contents. Large opacities involving a whole lung may be taken to indicate obstruction and possible perforation of the main bronchus, and should warrant bronchoscopy and consideration of early surgical intervention.

Bronchoscopy and bronchography are required before operation on any segmental lesion, in order $\omega$ to determine the stage of the disease process; and in some cases the bronchoscope may have a therapeutic application. Giraud and Métras (1953) emphasize the necessity of using a tube and sound in performing bronchography in these cases so as $\vec{\circ}$ to ensure that the contrast medium passes the bronchial obstruction. Tomography in these cases has pitfalls. In several of our cases cavitation in $\vec{c}$ segmental lesions was confidently diagnosed by $\mathbb{D}$ this method, but bronchography and examination of the resection specimens showed the "cavities" to be bronchiectatic in nature.

When operation has been decided upon it should $\vec{\oplus}$ be regarded as an exploratory procedure in the or first instance, as advocated by Dillwyn Thomas (1952). Only after direct study of the extent and relationship of the pulmonary and lymph-node lesions, with the knowledge of the stage of the disease gained by previous full investigation, is the $\frac{\otimes}{\otimes}$ surgeon in a position to decide on the best procedure, usually excision of lymph nodes or lobectomy or a combination of the two. It is to be remembered that the operation is one of election, not of absolute necessity, and technical difficulties should be approached accordingly.

\section{Chronic Pulmonary Tuberculosis}

This disease is comparatively rare in childhood, but is of importance because of its severity and $\delta$ high mortality at this period of life (Bentley and $₹$ others, 1954). While primary pulmonary tubercu-윽 losis generally follows a benign course in children of school age, chronic pulmonary tuberculosis is generally progressive, with a serious prognosis. Bentley and others (1954) surveyed 116 cases. treated during the years 1942 to 1946 , the average $N$ period of observation being six years. Forty-eight per cent. of the cases died, another $18 \%$ relapsed. Other authors have reported similar results (Price 1948 ; Widmer, 1952).

The relation of the disease to primary infection $\stackrel{?}{?}$ remains in doubt. Rich (1951) concludes that both exogenous and endogenous reinfection can and do occur. Endogenous reinfection is believed to arise ${ }_{\Omega}^{\circledR}$ mainly from the persistence of viable tubercle $\stackrel{\mathbb{Q}}{\Omega}$ bacilli in the mediastinal lymph-node component 
of the primary complex. In the present series, five of the 14 cases arose in close relation to a primary infection. Bentley and others (1954) suggested that lesions caused by primary infection, especially when situated in the vulnerable upper posterior parts of the lungs, may act as a starting-point for chronic pulmonary tuberculosis, provided that at the time when the individual reaches the period of susceptibility to this form of disease the lesions are still incompletely healed.

Cavitation is a prominent feature of chronic pulmonary tuberculosis in childhood and adolescence, and presents the main problem in the surgical treatment of the disease. Bentley and others (1954) observed cavitation in $55 \%$ of their moderately advanced cases and in $85 \%$ of their far advanced cases. These authors, however, point out that absence of cavitation does not imply a good prognosis, and that it should not carry much importance when decisions on surgical measures are being taken. No detailed studies on the effect of chemotherapy in this group of cases appear to have been published, but the clinical impressions of experienced observers (Levine, 1953) suggest that it profoundly influences the course and prognosis of the disease. The experience of the present series indicates that chemotherapy, as in adults, controls the soft, spreading lesions, leaving residual cavitation to be dealt with by collapse therapy or surgery.

The surgical treatment of cavitation in childhood has long been recognized as a difficult problem. Holmes Sellors (1944) reviewed the then available methods and concluded that thoracoplasty was contraindicated because of the resulting scoliosis and deformity, and that extrapleural pneumothorax and Monaldi cavity drainage had met with only limited success.

Continental authors regard extrapleural pneumothorax as a valuable measure. Vojtek and Sery (1952) reported 41 operations for extrapleural pneumolysis in 37 patients under 16 years of age with cavitated disease. There were two deaths from haemorrhage, two cases of nonspecific empyema, and one of bronchopleural fistula. In all cases the cavity appeared radiologically closed ; only two cases remained sputum positive. Speleostomy by the Maurer technique has had its advocates, but Catel (1952) reviewed 14 cases in which the operation was performed on children between 9 and 16 years of age, on the classical indication of a solitary cavity lying in the peripheral parts of the lung. The late results assessed 16 to 18 months after the conclusion of treatment were most unsatisfactory; only two cases could be classed as clinically cured, and nine were classed as unhealed with a hopeless prognosis.

Few published reports on resection for pulmonary tuberculosis make special mention of its application in childhood. Ross (1951) reported six pneumonectomies and seven lobectomies performed for "adult type pulmonary tuberculosis" in childhood. There were no deaths and immediate results were very good in all cases except one, and all the cases with positive sputum became negative. Rubin and Mishkin (1952) reported that 30 children between the ages of 7 and 16 years were subjected to thoracotomy for tuberculosis. Resection was carried out in 27 cases (of a segment in four, of a lobe in 19 , and of a lung in five). There were two operative deaths, 25 patients remained well, and only one had active tuberculosis after operation. Although classification was only briefly mentioned, most were undoubtedly cases of chronic pulmonary tuberculosis, although some had probably primary lesions, to judge by the descriptions given.

The results of resection in the present series (seven lobectomies, one operative death, six good results after an average observation period of two years) support the view that resection is the procedure of choice in these children. As in all the other published reports, the results have been excellent from the point of view of pulmonary function. The impairment of respiratory function which is sometimes a disadvantage of resection in adults does not arise in children ; all reports agree that even pneumonectomy is well tolerated.

The clinical indications are similar to those in adults, but also include cavitation in the upper and posterior parts of an upper lobe of a type which in an adult might be treated by thoracoplasty. In two cases of the present series the indication for lobectomy was a "tuberculoma" which appeared to be spreading. Kassowitz (1954) claims that in children resection of pulmonary tuberculoma is not indicated, and that the lesion generally undergoes involution and radiological "cure" under chemotherapy. On the other hand, Schaffner, Quinlan, and Hiltz (1954) have emphasized that such lesions generally communicate with a bronchus and are liable to spread at any time, so that they regard resection as the treatment of choice. In children there seems to be no doubt that resection should be undertaken if the tuberculoma fails to respond rapidly to conservative treatment.

Experience with extrapleural pneumolysis in the present series was not encouraging. Six 
operations yielded three poor results, and one of the good results was obtained in spite of inability to maintain the extrapleural pneumothorax. Two of the poor results had a technically excellent pneumothorax. In four of the six cases an apparently quiescent lesion in the opposite lung at the time of operation was the principal reason for employing this operation instead of resection. Baldry, Sumner, and Ward (1952) have shown that extrapleural pneumothorax in adults yields good results only in the limited number of cases where the lesion is confined to the upper lobe, is composed mainly of infiltration, and where any cavity is thin-walled and less than $3 \mathrm{~cm}$. in diameter. In view of the results obtained by Vojtek and Sery (1952) there would seem to be a place for the operation in children with bilateral disease, but the indications should be confined to those stated by Baldry and others. In the present series the poor results were probably due to employing the operation for large cavities and dense residual lesions. The work of Lowell and Conklin (1953) suggests that bilateral resection might be employed in cases with bilateral lesions unsuitable for extrapleural pneumolysis.

The operation of intrapleural pneumolysis was used once in the present series, but the patient developed a tuberculous empyema. The results of Rudström and Hedvall (1952) after an extensive trial of this operation with the advantages of modern technique and chemotherapy were most unfavourable.

\section{SUMMARY}

Twenty-nine cases of pulmonary tuberculosis in children under the age of 16 years treated by major surgical procedures have been reviewed. Fifteen cases were operated upon for primary lesions, 14 for chronic "adult type" lesions.

The literature relating to the pathogenesis and surgical pathology of pulmonary tuberculosis in children has been reviewed, and an attempt has been made to define the indications for operation on a pathological basis.

It is concluded that surgery has a place in the treatment of primary pulmonary tuberculosis in childhood. Up to the present it has been employed mainly for the removal of irretrievably damaged lung tissue, but with the aid of more accurate pathological diagnosis there is a prospect of employing surgery to prevent such damage. The indications for surgical treatment of chronic pulmonary tuberculosis in children and adolescents are much the same as in adults, but collapse operations have little place and in this age group resection is the method of choice.

I am indebted to Mr. Martin Fallon for permission to publish, to Mrs. Martin Fallon and Mr. Rento Hermann for translations, and to the chest physicians of the Region for access to their case records.

\section{REFERENCES}

Adler, D., and Richards, W. F. (1953). Thorax, 8, 223.

Baldry, P. E., Sumner, J. J., and Ward, R. L. (1952). Tubercle (Lond.), 33, 336.

Bentley, F. J., Grzybowski, S., and Benjamin, B. (1954). Tuberculosi in Childhood and Adolescence. NAPT, London.

Botelho, G. M. Chapchap, A., Pereira, H. L. G., and Cordeiro, O. V. (1951). Dis. Chest, $20,642$.

Boyd, G. L., and Wilkinson, F. R. (1954). Ibid., 2E, 442.

Boyd, G. L., and Wilkinson, F. R.

Brock, R. C. (1950). Thorax, 5, 5.
Burke, H. E. (1940). Amer. Rev. Tuberc., 42, 343.

Carlens, E., and Hellström, B. (1953). Acta paediat. (Stockh.), 42 43.

Catel, W. (1952). Z. Kinderheilk., 71, 497.

Daly, J. F., Brown, D. S., Lincoln, E. M., and Wilking, V. N. (1952) Dis. Chest, 22, 380

Dickey, L. B. (1952). Ibid., 21, 260.

Dickey, L. B. (1952). Ibid., 21, 260.

Eliasberg, H., and Neuland, W. (1920). Jb. Kinderheilk., 93, 88 - (1921). Ibid., 94, 102.

Engel, S. (1947). The Child's Lung. Arnold, London.

Giraud, P. and Métras, H. (1953). Rev. Tuberc. (Paris), 17, 437 Grégoire, M., Longefait, H., and Hartung, L. (1954). Pédiatrie, 9, 557.

Hutchison, J. H. (1951). Tubercle (Lond.), 32, 271.

Jeune, M., Bertoye, A., Mounier-Kuhn, P., and Beraud, C. (1952). Pédiatrie, 7, 763

Kassowitz, K. E. (1954). J. Pediat., 45, 153

Laff, H. I. Hurst, A., and Robinson, A. (1951). J. Amer. med. Ass., 146,778 .

Leorand $\mathbf{R}$ Razemon, Masure, and Soots (1952), Lille chir., 7, 85

Levine, M. I. (1953). J. Mich. med. Soc., 52, 1169.

Levitin, M., and Zelman, M. (1950). Amer.J. Dis. Child., 79, 30

Liaras and Houel, J. (1953). Moroc. méd., 32, 1021

Lowell, L. M., and Conklin, W. S. (1953). Amer. Rev. Tuberc., 68, 885 .

Lowys, P. and Joly, H. (1951). Rev. Tuberc. (Paris), 5 ser., 15, 738. Métras, H. (1952). Ibid.. 16, 569.

Oprenheimer, E. H. (1935). Bull. Johns Hopk. Hosp., 57, 247.

Podvinec, S., and Erak. P. (1951). Bronchoscopie, p. 75.

Price, D. S. (1948). Tuberculosis in Childhood, 2nd ed. Wright, Bristol.

Quinlan, J. J., Schaffner, V. D., and Hiltz, J. E. (1952). J. thorac. Surg. 23, 125

Rich. A. R. (1951). The Pathogenesis of Tuberculosis, 2nd ed. Thomas, Springfield, Illinois.

Ross, C. A. (1951). Thorax, 6, 375.

Rubin, M., and Mishkin, S. (1952), Surg. Gynec. Obstet., 95, 751. Rudström, P., and Hedvall, E. (1952). Acta tuberc. scand., 27, 38. Schaffner, V. D., Quinlan, J. J., and Hiltz, J. E. (1954). Canad. med. Ass. J., 70, 431 .

Sellors, T. Holmes (1944), Proc, roy. Soc. Med., 37, 588.

Semb, C. (1954). J. Oslo City Hosp., 4, 123.

Stevens, H. (1952). Proc. roy. Soc. Med., 45, 746

Thomas, Dillwyn (1952). Ibid., 45, 743.

Thomas, Dillwyn (1952). Ibid., 45, 743. Dis. Chest, 22, 46.

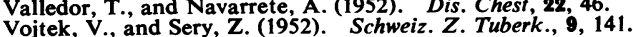

Vojtek, V., and Sery, Z. (1952). Schweiz.
Vossschulte, K. (1951). Chirurg, 22, 310.

Widmer, H. K. (1952). Schweiz. Z. Tuberk., 9, 147.

Williams, H., and Anderson, C. (1953). Quart. J. Med., 22, 295. 